Forty-six cases of ulcerative colitis were studied in detail. By comparison with Western series, ulcerative colitis as seen in this region is a milder disease with fewer complications and with less severe changes on sigmoidoscopy and rectal biopsy. The disease remains limited to the rectum, sigmoid colon, and descending colon in the majority of cases.

Owing to the high prevalence of specific infections of the colon there are difficulties associated with the diagnosis of ulcerative colitis, and these are discussed.

We are thankful to the Director-Principal of Maulana Azad Medical College and associated hospitals for permission to publish this work. We are indebted to Professor D. N. Gupta and Professor O. P. Bhardwaj for histological and radiological studies of the relevant material. We are grateful to Dr. J. B. Chatterjee, Dr. R. Subramanium, Dr. P. Raghvan, Colonel R. D. Ayyar, Dr. R. P. Malhotra, Dr. P. Koshy, Dr. P. N. Laha, and Dr. O. P. Gupta for assisting in the collection of information regarding the admission rate of ulcerative colitis. Thanks are also due to Dr. M. C. Watsa, Medical Department, Glaxo Laboratories Private Limited, for a liberal grant to carry out this study.

\section{REFERENCES}

Bacon, H. E. (1958). Ulcerutive Colitis. Philadelphia.

Bargen, J. A. (1930). Ann intern. Med., 3, 335.

Bebchuk, W., Rogers, A. G., and Downey, J. L. (1961). Gastroenterology, 40, 138.
Chopra, R. N., and Ray, P. N. (1939). Indian med. Gaz., 74, 65.

Craig, C. F. (1947). In Oxford Medicine, edited by H. A. Christian and J. Mackenzie. New York.

Daniels, G. E., O'Connor, J. F., Karush, A., Moses, L., Flood, C. A. and Lepore, M. (1962). Psychosom. Med., 24, 85.

Dennis, C., and Karlson, K. E. (1952). Surgery, 32, 892.

Edwards, F. C., and Truelove, S. C. (1963). Gut, 4, 299.

- (1964). Ibid., 5, 1 .

Felsen, J., and Gorenberg, H. (1936). Amer. 7. med. Sci., 192, 553.

Finch, S.' M., and Hess, J. H. (1962). Amer. F. Psychiat., 118, 819.

Fullerton, D. T., Kollar, E. J., and Caldwell, A. B. (1962). F. Amer med. Ass., 181, 463 .

Glenn, P. M., and Read, H. S. (1946). Gastroenterology, 6, 9.

Kirsner, J. B. (1959). F. Amer. med. Ass., 169, 433.

- Bicks, R. O., and Palmer, W L. (1957). Arch. intern. Med., 99. 642

and Goldgraber, M. B. (1960). Gastroenterology, 38, 536

McColl. I. (1956). Guy's Hosp. Rep., 105, 238.

Manson-Bahr, P. (1943). The Dysenteric Disorders, p. 433. London.

Matts, S. G. F. (1961). Quart. J. Med., 30, 393.

Melrose, A. G. (1955). Gastroenterology, 29, 1055.

Palmer, W. L. (1948). Ibid., 10, 767.

Pasricha, K. K., Chuttani, P. N., and Vidyasagar (1958). F. Ass. Phycns, India, 1,19 .

Powell, S. J., and Wilmont, A. J. (1966). Gut, 7, 438.

Rankin, F. 'W., Bargen, J. A., and Buie, L. A. (1932). The Colon, Rectum and Anus, p. 237. Philadelphia.

Ricketts, W. E., Kirsner, J. B., and Palmer, W. L. (1948). Gastroenterology, 10, 1.

and Palmer, W. L. (1946). Ibid., 7, 55.

Sloan, W. P., Bargen, J. A., and Gage, R. P. (1950). Ibid., 16, 25

Tandon, B. N.. Mathur, A. K., Mohapatra, L. N., Tandon, H. D., and Wig, Kं. L. (1965). Gra, 6, 448 .

Truelove, S. C. (1956). Brit. med. F., 2, 1267.

Virmani, P. (1963). Brit. 7. Surg., 50, 550.

Wilks, S., and Moxan, W. (1875). Cited by Bacon (1958).

\title{
Electroencephalogram and Retinal Vessels in Congenital Cyanotic Heart Disease Before and After Surgery
}

\author{
EVA M. KOHNER,* M.B., B.SC., M.R.C.P. ; E. M. ALLEN, † M.B., B.CHIR., M.R.C.P. \\ K. B. SAUNDERS,* M.D., M.R.C.P. ; V. M. EMERY $;$; C. PALLIS,§ D.M., F.R.C.P.
}

Brit. med.f., 1967, 4, 207-210

The electroencephalogram (E.E.G.) is often abnormal in patients with congenital cyanotic heart disease (Shev and Robinson, 1958 ; Lesny et al., 1960 ; Fowler et al., 1962 ; Allen et al., 1967). The reason for this is unknown. The disturbance of cerebral function might be due to structural brain disease, which is commonly present (Cohen, 1960), to chronic arterial desaturation, or to decreased cerebral blood flow resulting from a high packed cell volume (P.C.V.). Many of these patients also have abnormal fundi for which the polycythaemia has been incriminated (Taussig, 1960).

We have recently had the opportunity to study the retinal and E.E.G. changes in 12 patients with congenital cyanotic heart disease both before and at various intervals after operative treatment of their cardiac defects. The data obtained may throw some light on the cause of the abnormal E.E.G.s seen in these cases.

Angiographic study of the cerebral circulation in these patients is dangerous because of their known liability to cerebral thrombosis. The retinal vessels, however, are readily accessible for repeated examination, and their response to cardiac surgery may give some indication of what is happening at the level of the cerebral vasculature.

* Medical Registrar.

t Clinical Neurophysiologist.

t Clinical Neurophy

\& Senior Recordist.

Department of Medicine, Royal Postgraduate Medical School, London W.12.

\section{Patients and Methods}

Clinical and operative details concerning the 12 patients are given in Table I.

E.E.G. Studies.-All 12 patients had preoperative records. All except two (Cases 8 and 9) were again studied two weeks

Table I.-Clintcal Details of 12 Patients with Cyanotic Congenital Heart

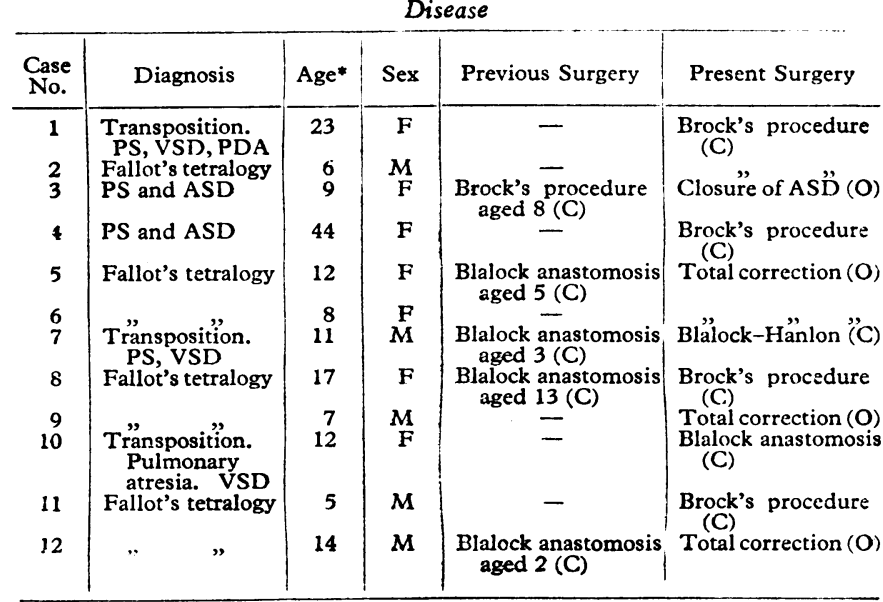

*At time of surgery. arteriosus. ASD = Atrial septal defect. $C=$ Closed surgical procedure. $O=O p e n$ procedure involving total body perfusion. 
postoperatively. Cases 1 to 9 (inclusive) were further investigated three months after the operation. In Cases 3, 12, and 9 further records were obtained at six months, 12 months, and three years respectively. Routine bipolar recordings were taken with a Kaiser 8-channel portable machine (T.R. 60), the international system of electrode placement being used.

Retinal Studies.-Retinal photographs were taken within 24 to 48 hours of the E.E.G. in all patients with the exception of one (Case 6), who was not studied, and of two (Cases 4 and 9) who had no pictures taken at three months. Case 8 was studied two weeks postoperatively as well. The pupils were dilated with $0.5 \%$ cyclopentolate and $10 \%$ phenylephrine. Retinal photographs were taken with a Zeiss fundus camera and Kodachrome II film. The disc, superior temporal, and macular areas were studied routinely. The prints were identified by code and allocated by three observers into one of four groups: normal or mildly, moderately, or severely abnormal. For purposes of grading, the following features were taken into account: degree of cyanosis of fundus, vascularity of disc, and calibre and tortuosity of retinal vessels.

\section{Results}

\section{Retinal Changes}

Preoperative.-In all the patients cyanosis gave the fundus a much darker hue than normal. The discs appeared more vascular than normal in Cases 1-4 and in Case 9. In Case 2 there was frank papilloedema (Fig. 1). In all patients both arterioles and venules were markedly dilated and tortuous. In addition there was a general increase in vascularity of the entire fundus, with many prominent small vessels. The excessive vascularization appeared to be due to normal, though dilated, arterioles and venules. These changes were most pronounced in the patients with severe or moderately severe cardiac disability. These were the patients with the highest P.C.V. (Table II).

Postoperative.-The greatest changes occurred during the first two weeks (the general condition of the patients precluded an earlier photographic assessment), and there was further improvement at three months. The fundi became much pinker (less cyanosed). Of interest was the development of " atrophic"

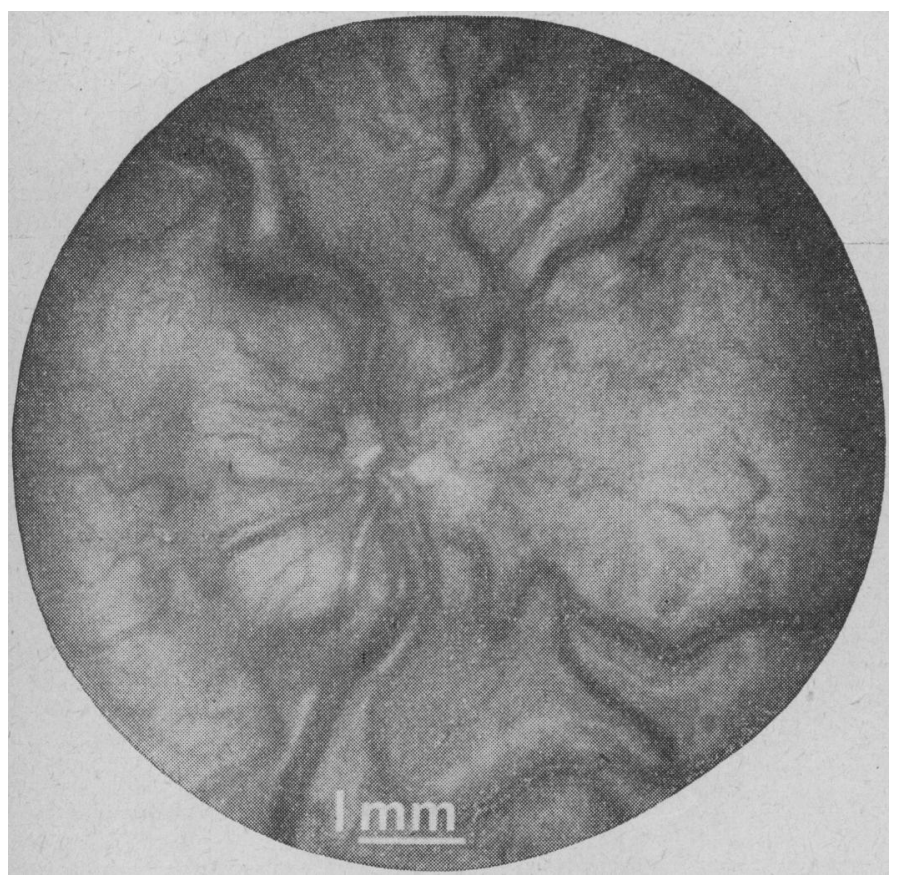

Fig. 1.-From a colour slide of Case 2, showing the disc area preoperatively. Note papilloedema, dilatation, and tortuosity of both arterioles and venules.
(" albinotic") fundi in three of the patients studied (Cases 3, 7, and 9). This may have been masked preoperatively by the engorgement of the retina and choroid.

The abnormal vascularity of the disc returned to normal in most instances. In the patient with papilloedema (Case 2) there was remarkable improvement, though the disc was still not quite normal at three months (Fig. 2).

The tortuosity and dilatation of the vessels decreased at two weeks and improved further at three months. The number of small vessels appeared to be reduced.

The greatest change in the fundal appearance (Case 2) occurred in the patient who also exhibited the greatest postoperative fall in the P.C.V. (from 80 to $44 \%$ ). The only patient whose fundal appearances remained unchanged at two weeks (Case 10) was found at operation to have a defect not amenable to correction. Her P.C.V. was not significantly affected by the operation.

\section{E.E.G. Changes}

(a) Preoperative.-Only two patients had normal records (Cases 7 and 9). In eight patients (Cases 1, 3-5, 8, and 10-12) the E.E.G. was unequivocally abnormal, showing various combinations of abnormally slow dominant rhythm, excess theta and delta activity, focal delta and sharp waves, and runs of spike and wave. The records of Cases 2 and 6 were doubtfully abnormal.

(b) Early Postoperative.-One record (Case 5) showed improvement: a reduction in spike and wave. This patient had been given anticonvulsant cover during the operation and was still receiving phenobarbitone. In one patient (Case 1) the record was unchanged. In the other eight patients the records had deteriorated. The deterioration usually consisted of a slower and more irregular dominant rhythm and/or increased theta and delta activity, usually bilateral.

(c) Late Postoperative.-Nine patients had records taken about three months after operation. Comparison with the preoperative records showed improvement in only three patients (Cases 1, 2, and 4). In these the alpha rhythm was more regular and there was a reduction of slower components. In two other patients (Cases 5 and 7) the E.E.G.s had reverted to their pre-

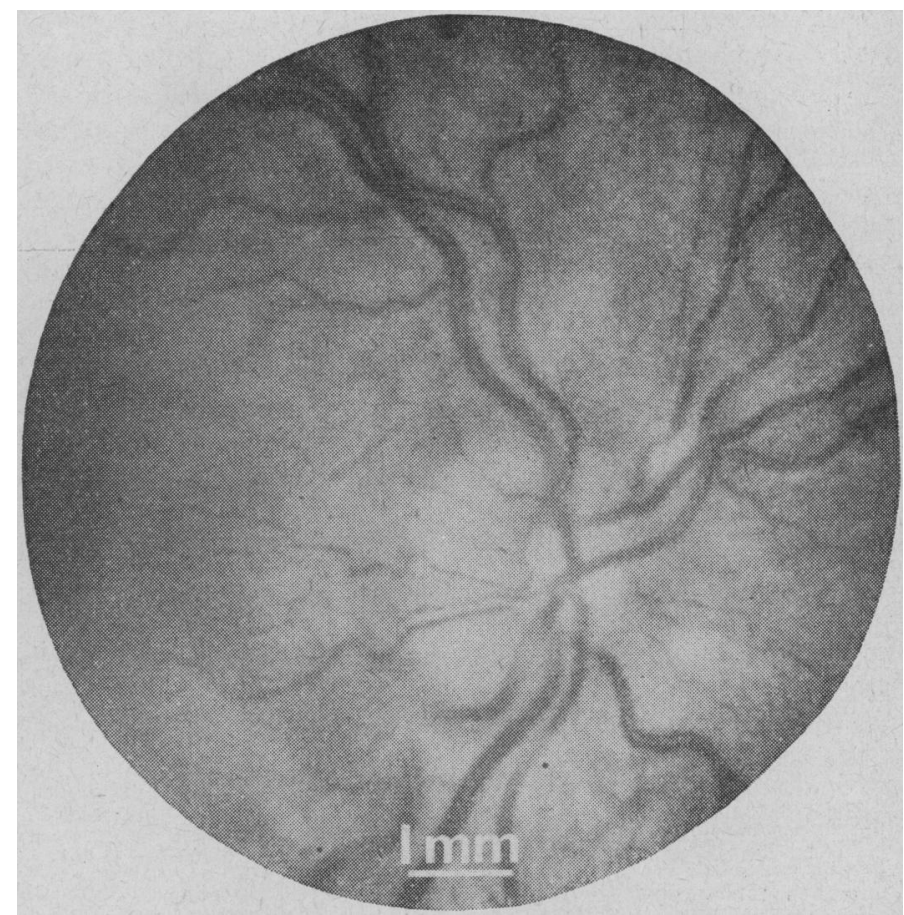

FlG. 2.-Same as Fig. 1. three months after operation. Disc margins almost normal Dilatation and turtuosity of vessels much less. 
operative pattern. In four patients (Cases 3,6, 8, and 9) the records were worse (slower and less rhythmic alpha activity and increased theta and delta activity). Fig. 3 illustrates the changes in a typical case.

Three years after operation the E.E.G. in Case 9 showed a return to the preoperative state.

Details concerning retinal appearances, E.E.G.s, and P.C.V.s are summarized in Table II.

\section{Discussion}

All patients had abnormal retinal vessels and a cyanosed fundus before operation. The abnormalities were most severe in the patients with the highest P.C.V., but it is probable that these patients also had the most severe arterial desaturation. The postoperative improvement in the appearance of the retinal vessels was associated with a fall in the P.C.V., but the arterial saturation had also reverted towards normal. The relative importance of a low $\mathrm{Po}_{3}$ and a high P.C.V. with increased blood viscosity in the causation of the retinal vascular changes were not ascertained in this study. Investigations of patients with hypoxia (and hypercapnoea) due to respiratory failure, a high haematocrit due to polycythaemia rubra vera, and a high plasma viscosity due to macroglobulinaemia are now in progress and may clarify this aspect of the problem.

The high incidence of abnormal E.E.G.s in congenital cyanotic heart disease has been confirmed. It is of interest that only two patients had clinical neurological abnormalities.
Case 1 had a history of past cerebral abscesses and still suffered from epilepsy. Case 2 had bilateral synergic movements ("mirror-movements") of the upper limbs and both clinical and radiological evidence of a craniovertebral anomaly.

No relation was established between the level of the P.C.V. and the degree of abnormality of the E.E.G.

The deterioration in six of the 10 E.E.G.s recorded two to three weeks postoperatively was unequivocal. One patient (Case 4) was thought to have suffered a postoperative cerebral embolus, but the other patients had been free from overt neurological complications. These early deteriorations in the E.E.G. occurred whether or not the patient had been perfused during the operation, and were often observed despite the fact that the patients had clinically improved.

Similar deterioration of the E.E.G. recorded some two weeks after surgery for congenital heart disease was reported in 16 out of 24 cases by Torres et al. (1959) and in 13 out of 36 cases by Fowler et al. (1962). Their data, however, are not strictly comparable to ours in that they refer to mixed groups, including both cyanotic and non-cyanotic cases. Torres et al. (1959) found no relation between the occurrence of abnormal postoperative E.E.G.s and the constancy or intermittency of the cyanosis, blood pressure readings during surgery, oxygen saturation measured during the surgical procedure, or the duration of extracorporeal circulation. Similar early and reversible deterioration in the E.E.G. was found in two "controls" who had uncomplicated cardiac operations without perfusion (resection of coarctation of the aorta and exploratory thoracotomy only). In a series of 24 children

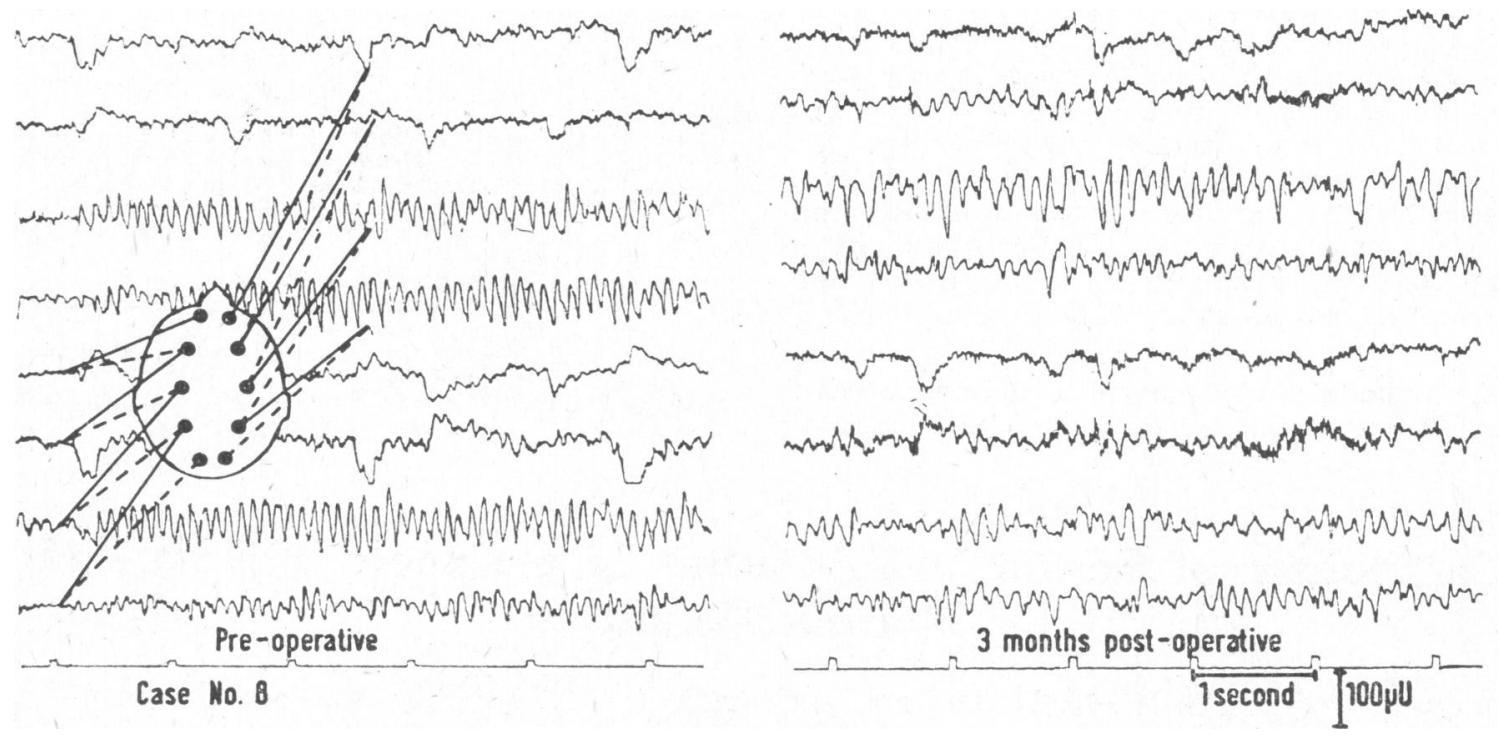

FIG. 3.-Case 8. E.E.G, records (preoperative and three months postoperative). Patient alert with eyes closed. Note postoperative slowing of alpha rhythm and increase of theta and delta activity, post-centrally.

TABLE II

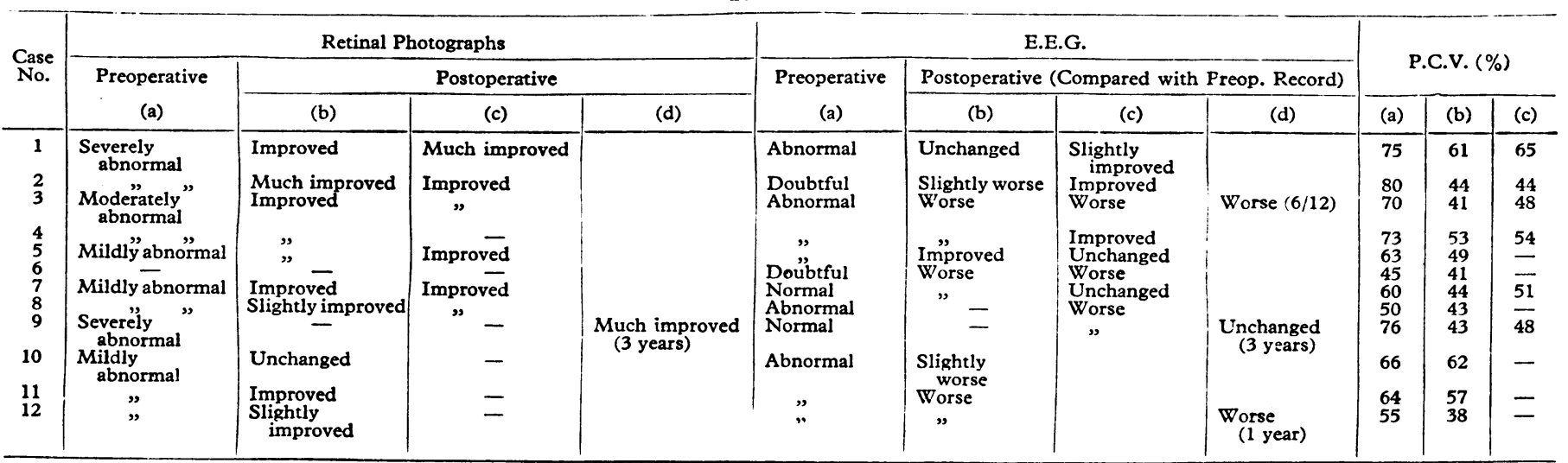

(a) Preoperative. (b) Two weeks postoperative. (c) Three months postoperative. (d) More than 3 months postoperative. 
submitted to various unspecified cardiothoracic operations Pampiglione (1965) found similar changes in the early postoperative E.E.G.s. These subsided within a week and were attributed to a metabolic response to trauma. In our patients the early deterioration lasted somewhat longer but was also usually reversible. In five of our eight patients with such early deterioration, records taken three months postoperatively had returned towards their preoperative state.

The deteriorated E.E.G.s at three months were more difficult to explain. In only two of our four patients where this occurred was any kind of explanation in fact forthcoming. One (Case 9) had a severe chest infection at the time of the record, and the other (Case 3) developed signs of congestive cardiac failure some time after the E.E.G. Though one patient (Case 6) developed unexplained ascites immediately after the operation, this had cleared by the time of the third E.E.G. We are also unable to explain why the record in Case 8 became worse. This patient had had "closed" heart surgery and had remained free from all complications four months postoperatively. A similar deterioration in the records of 9 out of 33 patients examined one to two years after surgery was found by Fowler et al. (1962). Long-term follow-up is obviously necessary to determine the significance of these findings.

In only three of our 10 patients having late postoperative E.E.G.s was surgical treatment of the cardiac lesion eventually followed by an improvement in the E.E.G., despite the fall in packed cell volume and the increase in oxygen saturation that resulted from the operation. In 20 out of 33 patients with abnormal preoperative E.E.G.s examined by Fowler et al. (1962) the record was unchanged one to two years after cardiac surgery. That initially abnormal records should remain abnormal for months or even years after operation is perhaps not surprising when one recalls the high incidence of microscopic abnormalities of the brain in patients with congenital heart disease (Cohen, 1960), even if not submitted to surgery. The effects of chronic hypoxia may outlast the hypoxia itself. It is not inconceivable that long-acting hypoxia may permanently impair the normal maturation of cerebral rhythms, the net result being that adults with congenital cyanotic heart disease, even if operated on, may still show E.E.G. patterns of juvenile type.

From these studies it would appear that either postoperative changes in the retinal vasculature are not paralleled by similar changes in the cerebral blood vessels, or that if changes in the cerebral vasculature do occur they are not followed by an improvement in the E.E.G., persistent abnormalities of which reflect other irreversible disturbances.

\section{Summary}

Retinal vascular changes are present in all cases of congenital cyanotic heart disease. The severity of these changes is most marked in patients with the highest P.C.V.s.

The E.E.G. is frequently abnormal in congenital cyanotic heart disease even in the absence of overt neurological symptoms and signs. The degree of E.E.G. abnormality bears no relation to the P.C.V.

The dramatic reversion towards normality shown in the retinal vessels after correction of the cardiac lesion is not paralleled by improvements in the E.E.G. In only a minority of cases is an improvement in the E.E.G. detectable three months after operative correction of the cardiac defect.

The E.E.G. defect in congenital cyanotic heart disease is not a simple functional disturbance directly related to arterial desaturation or to a diminution of cerebral blood flow from polycythaemia.

We would like to thank Professor J. F. Goodwin, Professor H. H. Bentall, and Dr. C. Oakley for allowing us to study patients under their care, and Dr. C. Dollery for helpful criticism.

E. M. K. was supported by the Tobacco Research Council and the photographic material was supplied by the Medical Research Council.

\section{REFERENCES}

Allen, E. M., Emery, V. M., and Pallis, C. (1967). In preparation.

Allen,

Fowler W. M. Kavan, E. M., and Walter, R. D. (1962). Amer. 7. Dis.

Child., 104, 131.
Lesny, I., Bor, I., and Vlach, V. (1960). Electroenceph. clin. Neurophysiol., 12. 534 .

Pampiglione, G. (1965). Lancet, 2, 263.

Shev, E. E., and Robinson, S. J. (1958). Electroenceph. clin. Neurophyszol., 10, 253 .

Taussig, H B. (1960). Congenital Malformations of the Heart, 2nd ed. Cambridge, Massachusetrs.

Torres, F., Frank, G. S., Cohen, M. M., Lillehei, C. W., and Kaspar, N. (1959). Neurology (Minneap.), 9, 174.
It has been shown that the half-life of serum $\gamma \mathrm{G}$-globulins is apparently characteristic of the species of origin (Spiegelberg and Weigle, 1965). However, it is also well established that the half-life value may be influenced by several factors, in particular by its dependence on the concentration of $\gamma \mathrm{G}$-globulin in the serum (Fahey and Robinson, 1963 ; Fahey and Sell, 1965).

Several workers have studied the catabolism of electrophoretically and antigenically distinct components of the YG-globulin of mice (Fahey and Sell, 1965 ; Tee, Watkins, and Wang, 1965). Each of these components was found to have a different half-life in the normal mouse. In view of the heterogeneity in biological functions of the antibodies associated with these components and the possible implications for immuno-

\footnotetext{
- Department of Pathology, King's College Hospital Medical School,
} London S.E.5. logical tumour enhancement (reviewed by Bloch, 1965), it is surprising that little significance appears to have been attached to the findings of those workers (Mills, Calkins, and Cohen, 1961) whose experiments indicate a similar catabolic heterogeneity of $\gamma \mathrm{G}$-globulins in man.

This paper reports some preliminary results of studies being made in this laboratory of the catabolic heterogeneity of human $\gamma \mathrm{G}$-globulins.

\section{Volunteers and Methods}

Two male volunteers were used in this study. The control subiect was one of us (J.W.) aged 31 . The other volunteer was a patient aged 43, who over the previous seven years had undergone surgery for the removal of six distinct primary carcinomas. In 1960 he had a laryngectomy for carcinoma of 